\title{
The TORCH time-of-flight detector
}

\author{
T. Blake ${ }^{* a}$, S. Bhasin ${ }^{b, c}$, N. H. Brook ${ }^{c}$, T. Conneely ${ }^{d}$, D. Cussans ${ }^{b}$, M. van Dijk ${ }^{e}$, \\ R. Forty ${ }^{e}$, C. Frei ${ }^{e}$, E. P. M. Gabriel ${ }^{f}$, R. Gao ${ }^{g}$, T. J. Gershon ${ }^{a}$, T. Gys ${ }^{e}$, \\ T. Hadavizadeh ${ }^{g}$, T. H. Hancock ${ }^{g}$, N. Harnew ${ }^{g}$, M. Kreps $^{a}$, J. Milnes ${ }^{d}$, D. Piedigrossi ${ }^{e}$, \\ J. Rademacker ${ }^{b}$
}

a Department of Physics, University of Warwick, Coventry, UK

${ }^{b} \mathrm{HH}$. Wills Physics Laboratory, University of Bristol, Bristol, UK

${ }^{c}$ University of Bath, Bath, UK

${ }^{d}$ Photek Ltd, St Leonards on Sea, UK

${ }^{e}$ European Organisation for Nuclear Research (CERN), Geneva, Switzerland

${ }^{f}$ School of Physics and Astronomy, University of Edinburgh, Edinburgh, UK

${ }^{g}$ Denys Wilkinson Laboratory, University of Oxford, Oxford, UK

E-mail: T.Blake.1@warwick.ac.uk

\begin{abstract}
The TORCH detector is a time-of-flight system that is being developed for use in particle physics experiments with the aim of providing particle identification, over a wide area, in the momentum range 2 to $10 \mathrm{GeV} / c$. The detector exploits prompt Cherenkov light produced by charge particles traversing a $10 \mathrm{~mm}$ thick quartz plate. Photons propagate via total-internal reflection and are focussed onto a detector plane comprising position-sensitive micro-channel plate photomultiplier (MCP-PMT) detectors. The goal is to achieve a resolution of $15 \mathrm{ps}$ per particle by combining information from around 30 detected photons, given a single-photon resolution of $70 \mathrm{ps}$. The MCP-PMT detectors have been developed with a commercial partner (Photek), leading to the delivery of a square tube with a 53-by-53 mm active area and 8-by-128 pixel equivalent. A smallscale TORCH demonstrator has been operated in beam tests and preliminary results indicate a single-photon resolution better than 100 ps. Progress towards a larger-scale system with 11 MCP-PMTs is presented.
\end{abstract}

The 39th International Conference on High Energy Physics (ICHEP2018)

4-11 July 2018

Seoul, Korea

${ }^{*}$ Speaker. 


\section{TORCH concept}

TORCH is a large-area time-of-flight detector, designed to provide particle identification (separation between charged pions, kaons and protons) in the range 2 to $10 \mathrm{GeV} / c$ [1]. The TORCH concept exploits the production of Cherenkov light by charged particles entering a quartz radiator plate. The photons are then transported to the periphery of the detector by total internal reflection within the plate, where they are focussed by a cylindrical focussing block onto an array of fast-timing photon detectors. To provide identification over the desired momentum range, a time resolution of $15 \mathrm{ps}$ per particle is required over a flight distance of $10 \mathrm{~m}$. This corresponds to a single photon resolution of $70 \mathrm{ps}$, given approximately 30 detected photons per charged particle.

An illustration of the layout of a possible full-scale $5 \times 6 \mathrm{~m}^{2}$ TORCH detector is shown in Fig. 1. Such a detector could upgrade the particle identification capabilities of the LHCb detector in the 2 to $10 \mathrm{GeV} / c$ momentum range. In the current detector, kaon, pion and proton separation is achieved using a pair of gaseous RICH detectors with $\mathrm{C}_{4} \mathrm{~F}_{10}$ and $\mathrm{CF}_{4}$ radiators [2]. At momenta less than $10 \mathrm{GeV} / c$, kaons and protons are below the Cherenkov threshold of the gas radiators.
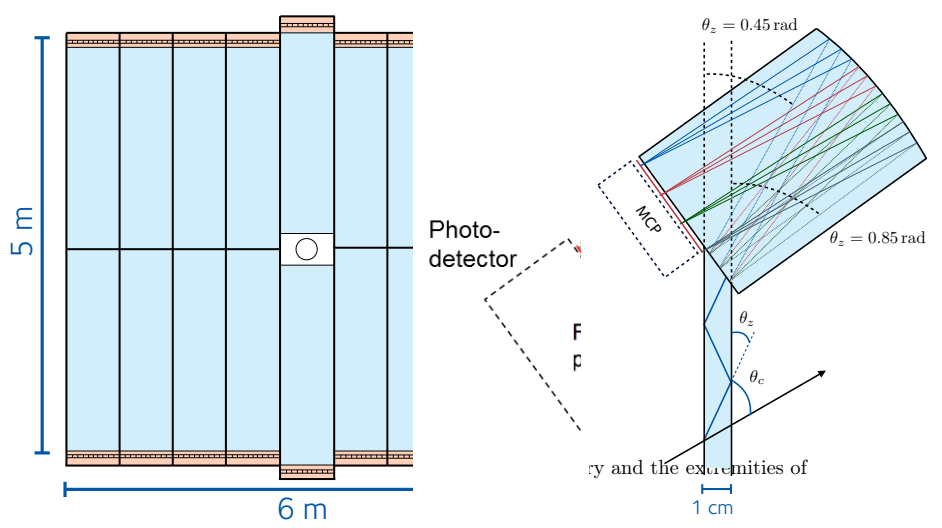

Figure 1: Illustration of a possible full-scale TORCH system utilising a $10 \mathrm{~mm}$ thick quartz radiator plate over an active area of $5 \times 6 \mathrm{~m}^{2}$. Cherenkov light produced by charged particles entering the radiator plate is transported to an array of fast-timing MCP-PMT detectors via total internal reflection and a cylindrical focussing block.

\section{MCP-PMTs}

Fast timing of photons is provided by an array of microchannel plate photomultipliers (MCPPMTs), developed as part of a three phase $R \& D$ programme with a commercial partner, Photek Ltd [4]. The MCP-PMTs provide a good intrinsic single photon time resolution of $\sim 35 \mathrm{ps}$ [3]. The aim of this programme is to develop a device that can be used in the challenging LHC environment, capable of withstanding a large integrated charge of $>5 \mathrm{Ccm}^{-2}$ on its anode. This is achieved through an atomic-layer-deposition coating [5]. The third phase (phase III) device is a multi-anode square tube with a $53 \times 53 \mathrm{~mm}^{2}$ active area. The 64-by-64 array of anodes is ganged to form either a 64-by-8 or a 64-by-4 pixel device. The effective pixelisation is increased by a further factor of two in the fine pixel direction by exploiting charge sharing between neighbouring pixels. The granularity of the detector is designed such that the photon angle in the vertical and horizontal 
planes can be measured to $1 \mathrm{mrad}$ in the TORCH. A photograph of the front and rear of a phase III prototype MCP-PMT is shown in Fig. 2. The pixel readout connectors are mounted on an external $\mathrm{PCB}$ and connected via anisotropic conductive film (ACF).
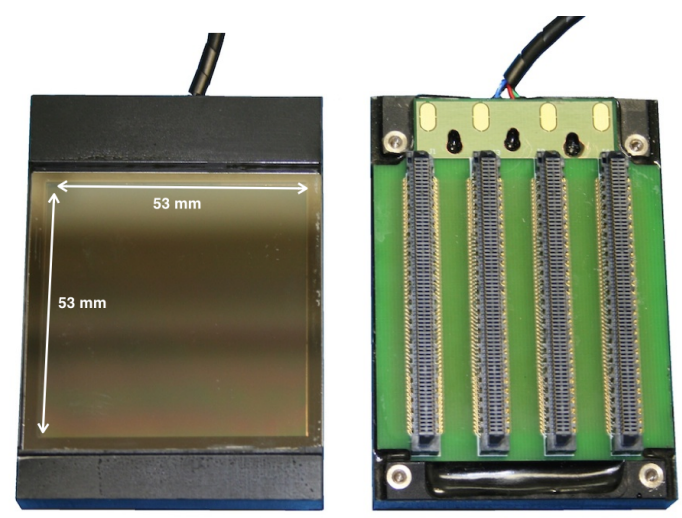

Figure 2: Phase III MCP-PMT with 64-by-8 pixels viewed from (left) the front and (right) behind. The MCP-PMT is designed to be close-packed in one direction with an active area greater than $80 \%$. Readout connectors for the eight pixel columns are mounted on an external PCB and connected via ACF.

\section{Test beam results}

A small-scale prototype of the TORCH detector has been operated in November 2017 and July 2018 in a $5 \mathrm{GeV} / c$ mixed $p / \pi^{+}$beam at the CERN PS. This prototype employs a quartz radiator plate with dimensions $120 \times 350 \times 10 \mathrm{~mm}^{3}$ and is instrumented with a single phase III MCP-PMT. The $p$ and $\pi^{+}$in the beam can be distinguished using two $\mathrm{CO}_{2}$ Cherenkov threshold counters located upstream of the TORCH detector. Two time reference signals are provided by a pair of borosilicate fingers, that are each instrumented with a single-channel MCP-PMT. The fingers are located approximately $10 \mathrm{~m}$ upstream and $1 \mathrm{~m}$ downstream of the TORCH prototype. The beam is also instrumented with a silicon pixel telescope to accurately determine the position and the direction of the $p / \pi^{+}$at the entrance to the radiator plate. The MCP-PMT is read out by a custom electronics system using the NINO and HPTDC readout chips developed for the ALICE experiment [6].

Figure 3 (left) shows the arrival time and vertical pixel coordinate of reconstructed photons in a single pixel column. The dataset was taken with the beam impinging close to the edge of the plate and $\sim 140 \mathrm{~mm}$ below its centre and has been pion-enriched using information from the Cherenkov threshold counters. The banding in the figure is characteristic of the TORCH configuration, with different bands corresponding to different numbers of reflections from the side faces of the radiator plate. A data-driven approach is used to correct for integral non-linearities and time-walk in the readout electronics [7]. The data are overlaid with the result of a simulation of the TORCH geometry. The difference between the observed and expected arrival time of the photons, $\Delta t$, is shown in Figure 3 (right). The $\Delta t$ distribution is well described by a Gaussian distribution with a power-law tail, and is indicative of a single photon resolution of $\sim 100 \mathrm{ps}$. The tail of the distribution is due to imperfect corrections to the photon arrival time. Improvement in the resolution is expected with an updated charge-to-width calibration of the readout electronics. 

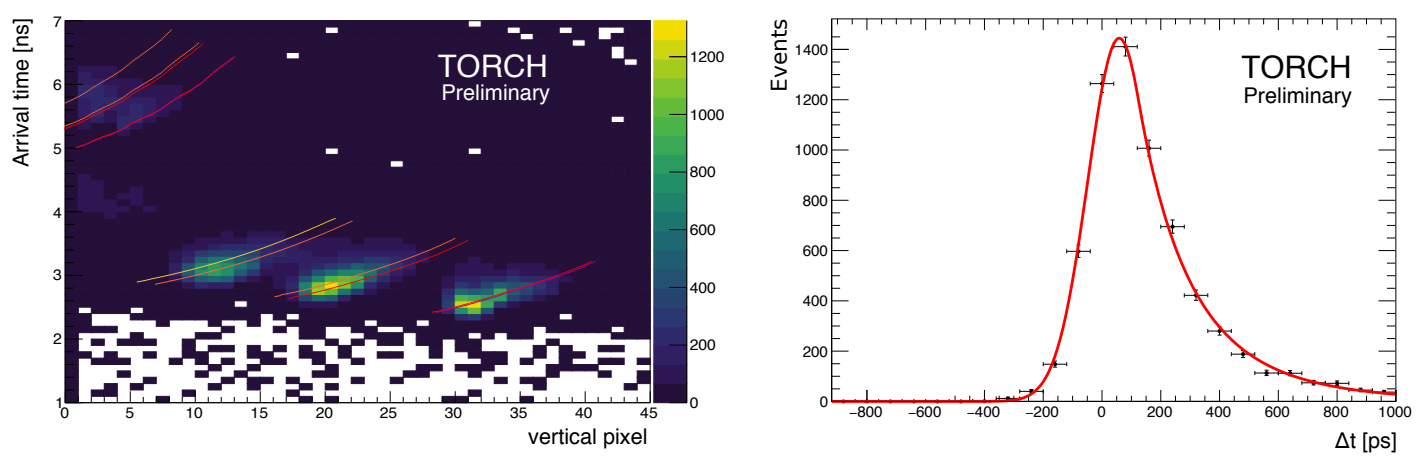

Figure 3: Vertical pixel and arrival time of reconstructed photons (left) and time residual (right). The lines in the left-hand figure indicate the result of a simulation of the TORCH prototype in a $5 \mathrm{GeV} / c \pi^{+}$beam. The time residuals are estimated by comparing the measured and predicted arrival times of the reconstructed photons.

A larger scale prototype detector, using a $660 \times 1250 \times 10 \mathrm{~mm}^{3}$ radiator plate, has been developed for use in a beam test at the CERN PS in October this year. This larger-scale system can be equipped with up to eleven 64-by-8 pixel phase III MCP-PMTs, read out by 44 NINO and 88 HPTDC boards, and will serve as a demonstrator for a full-scale TORCH module.

\section{Acknowledgments}

Support is acknowledged from the Science and Technology Research Council, UK, and the European Research Council (through the FP7 Advanced Grant ERC-2011-AdG 299175- TORCH). T. B. would like to acknowledge support from the Royal Society, UK.

\section{References}

[1] M. J. Charles and R. Forty, TORCH: Time of Flight Identification with Cherenkov Radiation, Nucl. Instrum. Meth. A 639 (2011) 173.

[2] M. Adinolfi et al., LHCb RICH collaboration, Performance of the LHCb RICH detector at the LHC, Eur. Phys. J. C 73 (2013) 2431.

[3] T. Gys et al., Performance and lifetime of micro-channel plate tubes for the TORCH detector, Nucl. Instrum. Meth. A 766, 171 (2014).

[4] T. M. Conneely et al., The TORCH PMT: a close packing, multi-anode, long life MCP-PMT for Cherenkov applications, JINST 10 (2015) C05003.

[5] T. M. Conneely, J. S. Milnes and J. Howorth, Extended lifetime MCP-PMTs: Characterisation and lifetime measurements of ALD coated microchannel plates, in a sealed photomultiplier tube, Nucl. Instrum. Meth. A 732 (2013) 388.

[6] R. Gao et al., Development of TORCH readout electronics for customised MCPs, JINST 11 (2016) C04012.

[7] N. Brook et al., Testbeam studies of a TORCH prototype detector, Nucl. Instrum. Meth. A 908 (2018) 256. 